\title{
IX.
}

\section{Ueber die Farbenbezeichnungen bei den römischen Dichtern ${ }^{1}$ ).}

Wir haben bei den Farbenbezeichnungen, von denen wir im Folgenden handeln wollen, im wesentlichen drei verschiedene

1) Ein zu der folgenden Abhandlung grehöriges Bruchstück, welches von den wesentlichsten Farbenbezeichnungen für Roth (ruber, rubicundus, rutilus etc.) handelt, habe ich in den Philologischen $\mathrm{Ab}$ handlungen, Martin Hertz zum 70. Geburtstage von ehemaligen Schülern dargebracht (Berlın 1888), S. 14 ff. veröffentlicht. Was das Material zu meiner Untersuchung anlangt, so ist dabei die heidnische Poesie voliständig in Betracht gezogen; von der christlichen, abgesehn von den was bei Baebrens Poetae Latini minores (von mir unter P. L. M. citiert) steht, noch die Gedichte des Ausonius (nach der Ausgabe von Peiper), Claudian (Jeep), A pollinaris Sidonius (Lütjohann), Martianus Capella (EyBenhardt) Corippus (Partsch). Mit A. L. ist die Anthologia Latina, Bd. IV der P. L. M., gemeint. Aeltere Litteratur über den hier behandelten Stoff giebt es wenig. In Betracht kommen vornehmlich folgende Abhandlungen: D $8 \mathrm{ring}$, de coloribus veterum. Gotha 1788 (auch in dessen Commentationes, Nürnberg 1839, S. 86 ff.) C. G. Jaco b, Quaestiones epicae, Lips. 1839 , p. $69 \mathrm{ff}$. Marg, de usu et significatione epithetorum quorundam colores indicantium, Bromberg. Gymn. Progr. v. 1857. O. W e is e, Die Farbenbezeichnungen bei den Griechen und Römern, im Philologus XLVI $593 \mathrm{ff}$. Ueber vergilische Farbenbezeichnungen handelt $\mathrm{Th}$. R Price im American Journal of philology IV $1 \mathrm{ff}$ (mir unzugänglich). $\mathrm{Vgl}$. auch noch B o e $\mathrm{hmer}$, De colorum nominibus equinorum, in dessen Romanischen Studien I 231 ff. und den Aufsatz von 0. W eise, Die Farbenbezeichnungen der Indogermanen, in $\mathrm{Bezzenbergers}$ Beiträgen z. Kunde der indogerman. Sprachen, II 273. Anderes wird bei Gelegenheit angetührt werden. (Ich verdanke den Nachweis verschiedener älterer Abhandlungen der Freundlichkeit meines verebrten Lehrers M. Hertz). Der kurze Abschnitt in G o e the s Farbenlehre: „Farbenbenennungen der Griechen und Römer" ist von Interesse, aber ohne Belegstellen. 
Arten zu (unterscheiden: 1) solche, welche absolut eine bestimmte Farbe in allen ihren Nüancen bezeichnen und nicht von der Vergleichung mit irgend welchem farbigen Gegenstande entlehnt sind, sondern an sich schon in ihrem Stamm die Bedeutung der Farbe enthalten. Das sind also, wie bei uns weiß, schwarz, roth etc., so im Lat. albus, ater, ruber u. dgl. 2) diejenigen, welche von einem Vergleich mit irgend welchem Gegenstand der belebten oder unbelebten Natur entnommen sind, wie unser rosig, lat. roseus, lacteus, oder auch einen Farbstoff bezeichnen, wie purpurn, purpureus, ohne daß dabei der Gedanke der Färbung durch diesen Stoff noch festgehalten wäre. Diese Ausdrücke gehören, wie bei uns so auch im Lateinischen, vornehmlich der poetischen Diction, weniger der Sprache des täglichen Lebens an; hinsichtlich ihrer Bildung ist zu beachten, daB in unserer leicht Zusammensetzungen bildenden Sprache, ebenso wie im Griechischen, die meisten solcher Farbenbezeichnungen den verglichenen Gegenstand entweder mit dem Namen der Farbe selbst verbinden: rosenroth, grasgrün, kohlschwarz, oder wenigstens die Endung

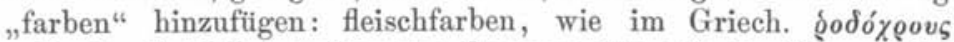
u. ä; der lateinischen Sprache dagegen, die sich der Composition viel weniger zu bedienen im Stande ist, genügt schon das vom verglichenen Gegenstande selbst gebildete Adjectiv mit der für Farben und Stoffe charakteristischen Endung eus, und zusammengesetzte Adjectiva mit color gehören erst der späten Latinität an. 3) Farbenbezeichnungen, die man in gewissem Sinne relative nennen kann. Streng genommen sollte man sie eigentlich überhaupt nicht Farbenbezeichnungen nennen, da sie weniger eine Farbe, als den Begriff der größeren oder geringeren Leuchtkraft oder Helligkeit, der Intensität irgend einer beliebigen Farbe enthalten, während sie freilich im Sprachgebrauch vornehmlich für die entsprechende Nüance einer bestimmten Farbe gebraucht werden. So bedeutet unser, ,blaß “ an und für sich jegliche Farbe in sehr zartem Ton, weshalb wir von blaßgelb, blabroth etc. sprechen; im speciellen aber verstehen wir darunter ein mattes weiß. Ebenso verhalten sich im Lat. candidus, pallidus, wahrscheinlich ursprünglich auch rutilus, indem dieselben an sich keine bestimmte weiße oder gelbe oder rothe Farbe sondern das Strahlende, das Blasse oder Stumpfe, das metallisch Leuchtende bedeuteten, aus dieser relativen Bedeutung aber, mehr und mehr in die absolute einer bestimmten, nur eben in der bezeichneten Weise nüancirten Farbe übergegangen sind.

Wir betrachten jedoch im Folgenden die Farbenbezeichnungen nicht nach den eben angeführten Unterschieden, sondern nehmen die Bedeutung der Farben selbst zum Eintheilungsgrund, indem wir mit Wei $B$ beginnen. 
I. Wei $B$.

1) albus $\left.{ }^{2}\right)$.

Während bei manchen Farbebezeichnungen, wie z. B. bei ruber (vgl. meine oben angefïhrte Abhandlung S. 16) im dichterischen Sprachgebrauch das Verbum des entsprechenden Stammes resp. das dazu gehörige Partic. praes. bei weitem häufiger zur Anwendung kommt, als das Adjectivum, gilt dies von albus keineswegs. Unter den ungefähr 300 Fällen, die für uns in Betracht kommen, fallen etwa 67 Proc. auf albus, 18 Proc. auf albere, davon 11 Proc. auf den Partic. albens, sodaß also das Adjectivum etwa sechsmal häufiger angewandt ist, als das Participium. Wie die Beispiele weiter unten zeigen werden, hat dabei albere viel häufiger die Bedeutung ,weißlich sein", als „weiß, d. h. von ansgesprochen weißer Farbe sein ". Von sonstigen zu dem Stamm gehörigen Wörtern kommt am häufigsten vor, obgleich im ganzen auch nicht zahlreich vẻrtreten, albescere (ich habe 17 Beispiele notirt); alle übrigen sind in unsrer poetischen Litteratur nur ganz spärlich vertreten: je einmal albidus (Ov. met. III 74); albulus (C atull 29, 8); albicascere (Matius ap. Gell. XV, 25); zweimal albatus (Hor. Sat. II 2, 61. Pers. 2, 40) und dreimal albicare (Catull. 63, 87. Hor. Carm. I 4, 4. Priap. 76, 2 Baehr.); dazu kommen noch einige vereinzelte Composita, nämlich albicapillus (Pla ut. Mil. gl. 631); albicomus (Venant. Fortun. 4, 2), albiplumis (Anth. Lat. 550, 11 Baehr.) und albicolor (Coripp. Iust. I 329).

Was nun die Bedeutung von albus ${ }^{3}$ ) anlangt, so bezeichnet es an sich die weiße Farbe $x \iota \tau^{\prime} ' \xi \xi o \chi \eta^{\prime} v$, namentlich im Gegensatz zur schwarzen; vgl. Lucr. II 820 : et quoniam plagae quoddam genus excipit in se Pupula, cum sentire colorem dicitur a $l b u \mathrm{~m}$, atque aliud porro, nigrum cum et cetera sentit. Catull. 93, 2: utrum sis albus an ater homo. Phaedr. III 15, 10: niger an albus nascerer. Der Gegensatz von albus zu dem ursprünglich der Klasse der relativen Farbenbezeichnungen angehörigen candidus, candere, wird sehr bestimmt von S e rvi us ad Verg. Georg. III 82 in folgenden Worten ausgesprochen: aliud est candidum, id est quadam nitenti luce perfusum esse, aliud al$b u m$, quod pallori constat esse vicinum. Diese Unterscheidung entspricht durchaus dem Sprachgebrauch: und der auch sonst ${ }^{4}$ )

2) Ueber albus und candidus handelt Marg a. a. 0. S. $12 \mathrm{ff}$.

3) Der von Döderlein, Lat. Synon. III 196 bezweifelte Zusam-

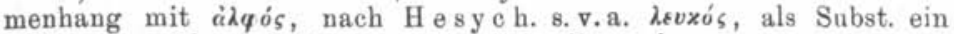
weißer Ausschlag, ist heut so ziemlich allgemein angenommen; vgl. Curtius, gr. Etymologie ${ }^{4}$, S. 292 f. K uhn, Ztschr. f. vgl. Sprachforschg. IV 109 .

4) Vgl. D o derlein a. a. O. 193. 
dafür angeführte Beleg V er g. Ecl. 7, 38: candidior cycnis, hedera formosior albà ist nach dieser Richtung hin ganz bezeichnend. In der That werden wir auch finden, daß auf gewisse Dinge, die eine sehr stark glänzende weiße Farbe haben, wie z. B. der Mond, albus niemals angewandt wird; für andere, welche ausgesprochen weiß, aber ebenfalls von starker Leuchtkraft sind, wie Schnee, Schwanenfedern u. dgl., kommt es zwar vor, aber bei weitem nicht so häufig, als candidus ${ }^{5}$ ). Ebenso charakteristisch ist die Anwendung beider Bezeichnungen auf die menschliche $\mathrm{H}$ a utfarbe. Für den weißen, aber dabei gesunden Teint von Frauen ist candidus ein gewöhnliches Attribut; hingegen kommt albus in solcher Anwendung nur ganz vereinzelt vor. Ein paar mal bedeutet es allerdings die von Natur weiße Hautfarbe, theils im Gegensatz zu derjenigen, welche durch Schminke erzielt wird (Hor. Sat. I 2, 124: nec magis alba velit, quam dat natura, videri), theils im Gegensatz zur schwarzen Race (I u v. $2,23)$; im Sinne von candidus, also wirklich als zartes, anmuthiges Weil, finden wir es nur Hor. Carm. II 5, 18 albo humero nitens; id. Sat. I 2, 36: mirator cunni albi; M a r t. XI 84, 3: alba brachia; $\mathrm{Claud}$. in Olyb. et Prob. 90: album pectus. Das sind aber die einzigen Stellen, welche man dafür anführen kann; eine verschwindend kleine Zahl gegenüber der Menge von Stellen, wo candidus in diesem Sinne steht. Sonst aber bedeutet albus, wo es von der menschlichen Haut gebraucht wird, nicht die natürliche gesunde Hautfarbe, sondern entweder die, welche durch Furcht, Schrecken u. dgl. entsteht (En n. trag. frg. 46 Vahl. Ov. am. I 7, 51. A p. Sid. 5, 601), daher auch direct albus timor, Pers. 3, 115 , oder pallor, Hor. ep. 7,15 ) ; oder es ist ein Zeichen von Krankbeit, zumal von Wassersucht (Hor. Carm. II 2, 15; Sat. II 2, 21. Pers. 3, 98. Sulpic. sat. 36. Seren. Sammon. 510; ähnlich ist wohl auch Plaut. Capt. 647 gemeint); oder es charakterisiert den sich den bräunenden Sonnenstrahlen nicht aussetzenden Städter (M a r t. I 55, 14; X 12, 9), daher auch übertragen M a r . III 58, 24: non segnis albo pallet otio copo.

Sehr häufig wird albus für die wei Ben $\mathrm{H}$ a a re des Alters gebraucht; zwar nicht so gewöhnlich, wie canus, grau, aber doch unvergleichlich öfter, als candidus. So finden wir es denn

5) Hingegen bat Doederlein ebenso unrecht, wenn er III 193 behauptet, daß das album sich mehr dem Gelblichen näbere, obschon das bi sweilen der Fall ist, wie wenn er VI 50 sagt, album sei das Weiße, insofern es der Gegensatz aller Farbe überhaupt ist, das Farblose, candidum aber das Weiße, insofern es selbst eine positive Farbe ist. Man vgl die Bemerkungen von Marg a. a. 0 ., welcher selbst den Satz aufstellt: Constat, omne candidum album quoque esse, non omne album etiam candidum, der allerdings nur theilweise zutreffend ist, da, wie die Beispiele oben zeigen, eine candida puella in der Regel nicht alba genannt werden kann.

Pinilologus. N. F. Bd. II, 1.

Brought to you by | University of Texas at El Paso 
bei crinis (I u v. 2, 112) und crines (V erg. A. VII 417 ; IX 651. $\mathrm{S}$ tat. Silv. IV 3, 116: alba crinibus); capillus (H o r. Carm. III 14, 25: albescens; id. ep. 17, 23. Pla ut. mil. gl. 631: albicapillus. Symphos. 188) und capilli (Ti b. I 8, 45. P r op. IV (III) 25, 13. Ov. her. 13161 ; met. XV 213: alba capillos. Priap. 76, 2 Baehr.: caput albicet capillis. M aximian. 2, $55)$; coma (O v. am. I 8, 111) und comae (O v. a. a. II 266; met. XIII 534; ex Pont. IV 12, 30, jedesmal albentes); caesaries (P. L. M. 19, I 44); barba (P l a u t. Bacch. 1101); auch direkt mit canities (O v. met. X 424) oder mit cani (Dra c ont. 8, 589: albentes cani, wo aber v. Duhn crines vermuthet) verbunden; ferner bei caput (N e mes. ecl. 1, 13), facies (M a r t. IV 78, 2: alba pilo), tempora (O v. met. III 516: albentia), und übertragen auch bei senecta ( $\mathrm{Prop}$. IV 4 (III 5), 24 und fast wörtlich gleichlautend $\mathrm{O}$ v. trist. IV 8, 2). - Wie in diesen Fällen nicht an silberweiße, sondern mehr an grauweiße Haare zu denken ist, so ist auch, wenn albus von $\mathrm{Z}$ ä $\mathrm{h} n$ e n gebraucht wird, wie P l a u t. Epid. 428, und von thierischen Zähnen V e rg. A. VII 667 ; XI 681, nicht der Glanz derselben, sondern die weiße Farbe an sich im Gegensatz zum umgebenden Mund oder thierischen Rachen die Hauptsache, wie die betr. Stellen das von selbst ergeben. - Häufig wird sodann albus resp. albere gebraucht von menschlichen oder thierischen Gebein en, bei denen ja auch die weiße Farbe in der Regel nur stumpf oder gelblich blaß ist; und zwar weniger in der Form, daß die Knochen selbst dies Attribut erhalten (so Hor. Sat. I 8, 16: albis informem ossibus agrum. Claud. rapt. Pros. III 341 [al. IV 10]: immania ossa . . . albent. A p. Sid earm. 7, 192: albentes lunae), als in der Weise, daß die Erde, auf der sie zahlreich liegen, als weils von Gebeinen bezeichnet wird: so campi, Ve $\mathrm{rg}$. A. XII 36 und in Nachahmung davon Coripp. Ioh. III 296; humus, Ov. Fast. I 558 und fast wörtlich ebenso III 708; scopuli, Verg. A V 865 ; solum Sen. Oed. 94 ; ef. S t at. Silv. II 7, 65: albos ossibus Italis Philippos. Uebrigens ist dabei noch hervorzuheben, daß es wohl nicht zufällig ist, wenn unter den zehn angeführten Stellen das Adj. albus nur dreimal vorkommt (V erg. A. V 865. Hor. u. Stat. 1l. 11.), sonst aber immer das Verbum albere (bei Coripp. 1.1. albescere) gebraucht ist; denn es liegt wohl darin der Gedanke, daß die Erde nicht an sich, sondern nur durch die auf ihr liegenden Gebeine weiß ist, weshalb man nicht alba humus direkt sagen wollte. Aehnlich sagt V a l. Fl. III 167: sparsusque cerebro albet ager. Das weiße Mark der Knochen erwähnt O v. met. XIV 207.

Was die weiße Farbe in der Thierwelt anlangt, so zeigen auch hier die Beispiele, daß albus zwar meist von reinem Wei $B$, vielfach aber auch von einem mehr in's Graue hinüberspielenden Weiß gebraucht wird. Unter den Vierfü Blern 
sind am häufigsten die $\mathrm{Pfer}$ e so bezeichneı, und zwar meist mit Beziehung auf den Triumph, bei welchem bekanntlich der siegreiche Feldherr mit einem Viergespann weiller Rosse auf das Capitol fuhr; vgl. Plaut. Asin. 279. V e rg. A. X 575; XII 164. Hor. S. I 7, 8. Prop. V (IV) 1, 32. Ov. met. VIII 33. Mart. VIII 26, 2. Claud. cons. Stilich. III 20. A p. Sid. carm. 2, 375. Nun sagt aber Verg. Geo. III 81 ff. von den Hengsten : honesti Spadices glaucique, color deterrimus albis Et gilvo. Wie man das zu verstehen habe, darüber waren schon die alten Erklärer nicht ganz einig. Servius giebt a. d. St. die oben angeführte Unterscheidung von candidus und albus, bemerkt aber weiterhin : multi ita legunt: "albis et gilvo", ut non album vel gilvum, sed albogiloum vituperet [quod falsum est]. quod si singuli colores vituperandi sunt, quan lo magis mixtus uterque, id est albogilvus? Indessen diese zweite Erklärung dürfte wohl schwerlich zu halten sein, da sonst Vergil sicherlich albis et gilvis geschrieben hätte; eher möchte man glauben, daß Virgil an dieser Stelle unter den equi albi die gewöhnlichen Schimmel versteht, deren Farbe allerdings mehr ein schmutziges Weiß ist, während man zu den Triumphalrossen nur tadellose Exemplare aussuchen mochte, wie solche bekanntlich in Persien besonders geziuchtet wurden. Sonst lieben aber die Dichter auch die weiße Farbe an gefleckten Rossen (Schecken) hervorzuheben, namentlich bei schwarzen Pferden: Verg. A. IX 49. Stat. Theb. VI 336. Corip p. Ioh. IV 520; und zumal in der Form, daß die Vorderfüße und die Stirn weiß sind, Verg. A. V 565: albis.... equus bicolor maculis, vestigia primi Albi pedis frontemque ostentans arcluus albam, und, vielleicht in Nachahmung, Sil. It. XVI 349 : patrium frons alba nitebat Insigne et patrio pes omnis concolor albo. Bei andern Vierfüßlern wird die weiße Farbe vornehmlich hervorgehoben, wenn es sich um Opferthiere handelt (alba victima, $\mathrm{O}$ v. Fast. I 720); so ganz besonders bei $\mathrm{R}$ in de r n (V e r g. Geo. II 146. Hor. C. saec. 49. Ov. ex P. IV 9, 50; auch wenn sonst etwas feierliches damit verbunden ist, wie $\mathrm{O}$ v. Fast. IV 826 ; am. III 4, 24; Erwähnung weiBgefleckter Rinder Verg. Geo. III 56). Ebenso kommt der weiße $\mathrm{Ziegen} \mathrm{bock} \mathrm{als}$ Opferthier vor bei Hor. C. III 8, 6; hingegen ist P. L. M. 19, I 7 der weiße Fleck auf der Stirn des Bockes eine besondere Schönheit, während bei V e r g. Ecl. 2, 41 : capreoli, sparsis etiamnunc pellibus albo, solche weiße Flecken gemeint sind, welche später, wenn die Thiere älter sind, dunkler werden, vgl. Serv. z. d. St. - Auch weiBe Lämmer als Opfer für oberirdische Gottheiten sind bekannt und oft erwähnt, V e r g. Geo. III 386 ; A. III 120. O v. Fast. I 56; hier galt die weiße Farbe ja nicht allein als Schönheit, sondern sie verlieh dem Thiere noch besondern Werth wegen der in reinem Weiß hochgeschätzten Wolle (vgl unten), daher Calpurn. ecl, 2, 36 den Gegensatz hervor- 
hebt: niger albae maritus ovis. Da man dem Wasser der Flüsse oder Bäche, von dem die Herden tranken, Einfluß auf die Färbung des Felles zuschrieb (vgl. Prisc. carm. 2, 431: hoc albat gurgite nigras), so nennt Mart. XII 63,3 den Galaesus, dem man auch solche Kraft nachrühmte, direkt albus. - Hingegen wird bei der weißen $\mathrm{S}$ a u mit ihren dreißig weißen Ferkeln in der bekannten Gründungsgeschichte von Alba longa die Farbe nur hervorgehoben, weil sie eben im Mythus eine Rolle spielt; s. Verg. A. III 392 ; VIII 45 u. 82. P rop. V (IV), 1, 35. I u v. 6, 177. (Zu Iuv. 13, 117: alba omenta porci bemerken die Erklärer alba e natura adipis; vel porci albi; doch ist wohl erstere Erklärung vorzuziehen). - Bei $\mathrm{Hunden}$ wird nur einmal von weißen Flecken im Fell gesprochen, $\mathrm{O}$. met. III 221. - Wenn albus als Attribut des Elephanten vorkommt (H o r. Ep. II 1, 196), so sind damit die seltenen weißen Elephanten gemeint, deren Farbe freilich auch kein ganz reines $\mathrm{Wei} B$ ist; wenn es dagegen mehrfach auch vom E sel gesagt ist $(\mathrm{O}$ v. met. XI 176: villis albentibus; $\mathrm{P}$ ers. 1, 59: auriculas albas), so ist hier direkt die Bedeutung graulich weiß anzunehmen. - Endlich können wir es hier noch anführen, daß der weißliche $\mathrm{S}$ ch a u m, welcher Thieren bei Anstrengung oder Wuth und wohl auch rasenden Menschen vor den Mund tritt, in der Regel albens genannt wird, seltner albus oder albidus (so Enn. Ann. 507. O v. met. III 74): bei Pferden $O$ v. met. XV 519. St at. Theb. VI 419. Enn. 1. 1., bei Hunden $\mathrm{O}_{\mathrm{v}}$ met. VII 415 ; bei Schlangen $O v$. met. III 74 ; bei einem rasenden Menschen Sil. Ital. IV 251.

Unter den V ögeln sind es die S chw än e, die am häufigsten dies Attribut erhalten, alhi olores, V e r g. A. XI 580. Ov. her. 7, 2. Stat. Theb. IX 858. Sil. It. XIV 190; albi cygni, Ov. met. XIV 519 ; der Schwan ist daher auch der weiße

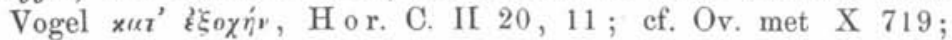
XII 144. Demnächst tritt albus zur $\mathrm{T}$ a u be, namentlich der der Venus heiligen, Tib. I 7, 18. Ov. Fast. I 452. Ps. Ov. her. 15, 37; ef. den albulus columbus bei Cat. 29, 8, und A nth. L. 550,11 (Baehr.): albiplumem columbam; ferner zur Gans, Hor. S. H 8, 88. Petron c. 93 v. 4 . Dagegen ist derwei Be $\mathrm{R}$ abe bei den Alten bereits ebenso sprichwörtlich als Seltenheit, wie bei uns der weiße Sperling; vgl. L u c r. II 822 : conveniebat enim corvos quoque saepe volantis Ex albis album pinnis iactare colorem. I u v. 7, 202: corvo rarior albo; und wenn I u v. 13, 141 von weißen Hennen spricht, so hat das den gleichen Sinn, da die italischen Landwirthe solehe weiße Hennen nicht liebten (Col u m VIII 2, 7) und dieselben daher ungewöhnlich waren. Auch sei hier noch bemerkt, daß die Excremente der Vögel als weiß bezeichnet werden; so vom Raben Hor. S. I 8, 37; vom Hahn Seren. Samm. 714. - Aus der übrigen Thierwelt 
sind keine Beispiele anzuführen, als höchstens der bei $\mathrm{Ov}$. met. VI 380 beschriebene weilse Bauch des Frosches.

Wenn wir zum Pflanzenreich übergehen, so sind es unter den Blumen selbstverständlich die Lilien, welche am häufigsten mit dem fast Epitheton perpetuum gewordenen albus versehen werden: Verg. Geo. IV 130; Aen. XII 68. Ps. Tib. III 4, 33. Prop. II 3, 10. Ov. fast. IV 442. Petron. c. 127 v. 5. Val. Fl. VI 492. Draeont. 6, 7. Sonstige Blumen erhalten das Attribut nur vereinzelt: so die überhaupt sehr selten erwähnten weißen Rosen (rosae albentes), Ov. a. a. III 182. A. L. 499, 6; die äußere Blattreihe der Narzisse (Ov. met. III 510), die weiße Kamille (Cat. 61, 190: alba parthenice), die Blüthe des Ligusters (Verg. Ecl. 2, 18. Claud. rapt. Pros. II 130) und des Birnbaums (Verg. Geo. II 81). Unter den Sträuchern können wir von vorn herein diejenigen aussondern, welche das Attribut albus mehr aus botanischem, als aus poetischem Gesichtspunkt erhalten, insofern es nämlich zur Bezeichnung der Gattung erforderlich ist: so bei der $\mathrm{Z}$ a un$\mathrm{r}$ ï be, bei den Alten vitis alba genannt, Ov. met. XIII 800 . Colum. X 347 ; ferner beim We i Bd orn, spina alba, Ov. Fast. VI 130 u. 165, und auch bei der einen Art des Epheu, hedera alba, Verg. Eel. 7,38, denn es gab auch eine hedera nigra, und Servius sagt $\mathrm{z}$. d. St.: nigra autem vel alba hedera non ex foliis sed ex ligno cognoscitur. Dasselbe ist auch Colum. X 417 mit der alba ficus der Fall, quae servat flavae cognomine cerae; der didaktische Dichter umschreibt auf diese Weise nur diejenige Feigenart, welche sonst albicerata hieß, s. Plin. XV 70 . Auch bei der Wei B pappel, populus alba, ist, wenn sie bei Dichtern so genannt wird, das Epitheton bisweilen ein lediglich botanisches, so namentlich bei Seren. Samm. 164 u. 697 ; allein in anderen Erwähnungen ist doch meist das Epitheton als ein wirklich bezeichnendes aus poetischen Rücksichten gewählt, da das hellere Laub der Pappel, das freilich noch ziemlich weit davon entfernt ist, rein weiß zu sein, damit charakterisiert werden soll; $\operatorname{man} v$ gl., abgesehen von bloßen Erwähnungen wie Hor. C. II 3, 9. Tib. I 4, 30 (wo die Hss. allerdings alta haben, aber alba eine durchaus wahrscheinliche Emendation ist). Ov. her. 9, 64, namentlich Stat. Silv. III 1, 185: populeaque movens albentia tempora silva, und Sil. It. X 531: albae populus alta comae. Wahrscheinlich hat man daher auch bei Val. Fl. V 10: pars auguris alba Fronde caput vittisque legunt die Blätter der Weißpappel zu verstehen; denn für die grauweißen Blätter der Olive kommt albus nur ganz vereinzelt vor $(\mathrm{O}$ v. her. 11, 67: ramis albentis olivae). Lucil. frg. $1181 \mathrm{k}$ (Lachm.) nennt auch den jungen Rebenschößling (pampinus) alba; der Farbenton desselben kommt ja auch in der That dem Weiß recht nahe. Wenn aber $\mathrm{O}_{\mathrm{v}}$. Fast. V 357 sagt: maturis albescit messis aristis, so ist 
da nach unserer Anschauung der Begriff des albus schon beträchtlich erweitert, da die reife $\mathrm{Halmfrucht}$ bei weitem mehr dem Gelb angehört, wie denn sonst auch flavus das gewöhnliche Attribut dafür ist. Es gehört das also zu den Fällen, wo albus in den Begriff des gelblich-weißen übergegangen ist; eben dahin rechne ich Calpurn. ecl. 4, 116: messis - nec inertibus albet avenis, vom wilden oder tauben $\mathrm{H}$ a fer gesagt. - Eßbare weiße $\mathrm{S}$ chwämme nennt Ov. Fast. IV 697.

Spärlich ist die Anwendung von albus für Objekte aus dem Mineralreich. Der Marmor, welchen die Griechen bekannt-

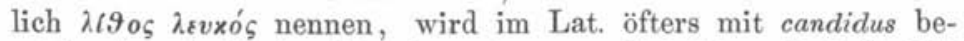
zeichnet; als lapis albus kommt er nur bei Hor. S. I 6, 116 und A p. Sid. carm. 11, 19 (vom parischen Marmor) vor. Plumbum album bei Lucr. VI 1077 ist der stehende Name für Z i n n, im Gegensatz zu plumbum nigrum, Blei (vgl. meine Technologie IV 81); alba cerussa, Bleiweiß (als Schminke), hat Mart. X 22, 2. Bei Verg. A. XII 87 wird album orichalcum genannt. Da man unter orichalcum später in der Regel Messing verstand (s. Technologie IV $194 \mathrm{ff}$.), so fiel das Attribut album bereits dem Servius auf, welcher z. d. St. bemerkt: alboque orichalco: auri scilicet comparatione; nam album non est; also nur im Vergleich zu dem gelben Golde werde das orichalcum weiß genannt. Falls nicht Vergil eine in der That weiße Erzmischung

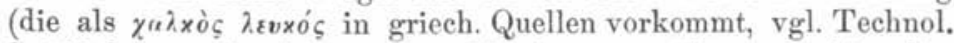
S. $198 \mathrm{fg}$.) gemeint hat, sondern Messing, so müBten wir hier diejenige Bedeutung von albus annehmen, der wir bisher noch nicht begegnet sind, die wir aber in andern Beispielen noch wiederfinden werden, nämlich h ell, wobei der Begriff der weißen Farbe in den Hintergrund tritt und der der Helligkeit, wenn auch nicht gerade im Sinn des Strahlenden, vorwaltet. - Sodann spricht Catull, 63, 87 von unida alhicantis loca litoris. Die Erklärer deuten das verschieden: die älteren denken an den weiBen Sand des Strandes (man könnte auch an weiße Uferfelsen denken); Riese erklärt: „glänzend von der Sonne beschienen“, was nicht gut angeht, da der Begriff des Glanzes dem albus fern liegt; Ellis und Baehrens fassen es als weiß vom Schaume der den Strand bespülenden Wogen, was am meisten für sich hat, da, wie wir unten sehen werden, gerade der Schaum des Meeres häufig durch albere bezeichnet wird. - Bei I u v. 1, 111 gehen die albi pedes des Emporkömmlings, der früher Sklave gewesen war, auf die Kreide, mit der man die Füße des zum Verkauf auf dem Gerüst aufgestellten Sklaven bestrich.

Wenden wir uns zu den Naturprodukten, so haben wir da zunächst der Wolle zu gedenken, an der die Dichter gern die weiBe Farbe hervorbeben, namentlich wenn es sich um den Gegensatz zur gefärbten handelt, so alba lana, Verg. Geo. II 465 , oder albens, Sil. It. XVI 569 ; album vellus, Ov. her. 
7, 100. Stat. Silv. I 2, 21, oder stamen, I u v. 12,65. Weiterhin ist zu nennen die $\mathrm{Mileh}, \mathrm{Ov}$. am. III 5, 13: (lac) quod spumis stridentibus albet (vgl. damit Priscian. carm. 2, 452 vom Steine Galaktit: albescit lacta liquescens), auch der Käse (Dracont. 8, 415: caseus albens); ferner EiweiB (Hor. S. II 4, 13) und Talg (Auson. XVIII 14, 19). Beim Wachs (Ov. am. I 12, 30. P. L. M. 42, VI 8) hat man natürlich nur an gebleichtes, nicht an Wachs im ursprïnglichen Zustande zu denken, da letzteres gelblich ist, s. unter flavus. Von weißem Pfeffer spricht Hor. S. II 4, 74 u. 8, 48; weiße Gra u pe nennt Stat, Silv. IV 9, 35. Albere kommt vereinzelt von $\mathrm{Per}$ len vor (A uson. X 70; albus lapillus bei A p. Sid. carm. 14, 3); bei Martial heißt es vom Elf enbein VII 13, 1 albescit, und VIII 28,12 albet, es bezieht sich dies aber nicht auf die natüliche Weiße desselben (hierfür ziehen die Dichter candidum ebur vor), sondern auf das Bleichen des gelbgewordenen (vgl. Mart. IV 61 mit der Anm. Friedlaenders). - Wenn in allen diesen Fällen der Begriff der Weiße mehr oder weniger festgehalten ist, so ist das dagegen nicht der Fall, wenn der Römer den hellen W ein, wie wir, weils nennt, vinum album, Plaut. Menaech. 915 ; Coum album, H o r. S. II 4, 29 ; Mareotides albae, Verg. Geo. II 91 (wenn Cor. Iustin. III 99 die dona Lyaei alba colore nivis nennt, so ist das eine arge poetische Uebertreibung). Hierbei ist wiederum der Begriff des Hellen maßgebend gewesen, namentlich im Gegensatz zum dunkeln Wein, den die Römer (wie heut noch die Italiener) schwarz (vinum atrum oder nigrum) nennen.

Auch im übrigen Gebiet der $\mathrm{Natur}$ und Naturerscheinungen findet albus bei den Dichtern häufige Anwendung. Vor allem haben wir da des Meeres zu gedenken. Dasselbe ist allerdings an und für sich, wenn es in Ruhe ist, kein mare album, sondern, wie wir an anderen Stellen sehen werden, caeruleum, bläulich; aber wenn Sturm es aufregt und die Wellen schäumenden Gischt aufwerfen, dann nimmt es jene Färbung an, welche zwar in der Regel bei den Dichtern mit canus, da sie sich mehr dem Grau nähert, aber auch häufig mit albus bezeichnet wird. Das Adj. albus selbst wird allerdings nirgends für diesen Zustand des aufgeregten Meeres gebraucht; die gewöhnliche Bezeichnung ist vielmehr das albescere, das weißlich-grauwerden durch den Schaum (V e rg. Geo. III 237: fluctus uti medio coepit cum albescere ponto, und fast wörtlich ebenso Aen. VII 528; ähnlich $\mathrm{O} v$. met XI 480: cum mare sub noctem tumidis albescere coepit fluctibus; ferner Lucr. II 773. Sil. It. VIII 429 u. XIV 360), oder albere, namentlich wenn dabei der Schaum, welcher dem Meere das geschilderte Aussehen verleiht, genannt wird (O v. met. VII 263: spumis tumentibus albet; ib. XI 501: spumis sonantibus albet; vgl. A p. Sid. carm. V 241: albet aquosa 
acies, nämlich von Seeungeheuern). Etwas anderes ist es, wenn Stat. Silv. I 3, 65 von albentes lacus in der Villa des Manlius Vopiscus spricht, womit wohl nur ,helle Teiche" gemeint sind, obgleich ich sonst keine Parallele hierzu anführen kann. Dagegen ist es wiederum gewöhnlich, daß die $8 \mathrm{chwefelhalti-}$ gen Gew ässer oder Quellen, die ein milchig-weißes Aussehen haben, durch albus näher charakterisirt werden, wie der Nar, Verg. A. VII 517: sulfurea Nar albus aqua. Sil. It. VIII 453: Nar. . . albescentibus undis. Claud. de VI cons. Hon. כૅ19: amnis... albet. - Weiterhin finden wir dann albus wieder in der Bedeutung des reinen Weiß, wenn es, was allerdings nur sehr selten der Fall ist, vom Schnee gesagt wird (Lucr. VI 736. Manil. Astr. II 419. M art. IV 2,6) oder vom $\mathrm{Hagel}$ (V arr. Sat. Menipp. p. 234, 5 Riese) oder vom Reif (H or. C. I 4, 4: nec prata canis albicant pruinis. A. L. 138, 20: humus hibernis albescit operta pruinis; cf. Coripp. Ioh. II 19), in welchen Fällen sonst häufiger canus vorkommt. Auch hier bemerken wir, daß albere, albescere, albicare gesagt ist, sobald das WeiBsein nicht vom Schnee oder Reif selbst, sondern von der damit bedeckten Erde ausgesagt ist (vgl. oben S. 146). Ebenso sagt Claud. rapt. Pros. III 232: rore albet ager, vom $\mathrm{Th}$ a in nicht gerade sehr passender Anwendung, da für blitzende Thautropfen der color albus nicht sehr angebracht scheint.

Zwischen den Bedeutungen grau und hell, die wir beide bereits mehrfach für albus gefunden haben, steht es gewissermaßen in der Mitte, wenn albus oder albescere, wie bekanntlich auch in Prosa sehr gewöhnlich, zur Bezeichnung der Morgendämmerung gebraucht wird (heut noch ital. alba, franz. aube). Auch wir sagen von dieser Morgenstunde sowohl "der Tag graut", als „es wird hell". So sprechen denn auch die Dichter von alba lux (Lucan. II 720) oder albens lux Coripp. Ioh. VII 84), lux albescit (V e r g. A. IV 586 (vgl. M a ti us ap. G e 11. XV 25: cum albicascit Phoebus) oder auch von den im Morgengrauen heller werdenden Gegenständen: von der Erde (V a l. F1. II 72: albet ager) oder von Bawwerken (id III 258: orta dies notaeque albescere turres). Damit hängt es zusammen, daB auch der Morgenstern dies Attribut erhält; Ovid spricht mehrfach von dem albus equus des Lucifer (met. XV 189 u. trist. III 5, 56). Auffallender ist Verg. Geo. I 365 ff. : saepe etiam stellas, vento inpendente, videbis Praecipitis caelo labi, noctisque per umbram Flammarum longos a tergo albescere tractus; der feurige Streif der Sternschnuppe scheint durch albescere nicht sehr treffend bezeichnet, doch deutet uns das noctis per umbram an, daB nicht so wohl an starken Glanz, als an den Gegensatz des hellen Streifens zu dem schwarzen Nachthimmel zu denken ist. Horat. C. I 12, 27 nennt auch das Sternbild der Zwillinge alba stella: vielleicht weniger im Sinne von hell, oder 
wie manche zu erklären meinen, in der unten noch zu erwähnenden Bedeutung günstig, glückbringend, als im Sinne von hellmachend, weil ihr Aufgang klaren Himmel und Ende der Sturmzeit bedeutet: also im gleichen Sinn, wie er auch diejenigen Winde, welche wolkenlosen Himmel bringen, albi nennt, den Iapyx, C. III 27, 19, und den Notus, der sonst als regenbringender Wind eher ater heißt, aber doch bisweilen, wie in den Bergen der Föhn, die Wolken vertreibt und klares Wetter bringt, C. I 7, 15: Albus ut obscuro deterget nubila coelo Saepe Notus neque parturit imbres Perpetuo $\left.{ }^{6}\right)$. - Geradezu hell, klar, bedeutet albus, wenn es von der Sonne gesagt ist, zumal im Gegensatz zur dunkeln Nacht; so Ennius wiederholt, Ann. frg. 92: sol albus recessit in infera noctis; fr. 547 : fugit albus iubar Hyperionis. Im gleichen Sinne wird der A ether, d. h. der klare Himmel, albus genannt, Catull. 63, 40. Sil. It. V 283; und ebenso die hellen, wolkenlosen Tage, wie sie der Hochsommer zu bringen pflegt, Mart. X 62,6 .

Unter den Produkten des GewerbfleiBes ist es vor allem die Kleidung, zu welcher sehr häufig die Bezeichnung albus hinzutritt, und zwar meist im Adjectiv (alba, Neutr plur., bedeutet direkt weiße Kleider, Ov. a. a. III 191 u. Fast. IV 619 ), welches auch an sich in poetischer Redeweise einen weißgekleideten bedeuten kann (P ers. 1, 16); letzteres wird bisweilen auch durch albatus wiedergegeben (Hor. S. II 2, 60. Pers. 2, 40). Seltner ist albens (Stat. Silv. V 2, 67. Corip p. Iust. II 316 ; von Binden Ov. met. II 410 u. XV 676); spät albicolor (Coripp. Iust. I 329). In den weitaus meisten Fällen der Erwähnung bezieht sich die Beifügung der weißen Farbe darauf, daß das festliche Tracht ist (vgl. die angef. Stellen und $\mathrm{O}_{\mathrm{v}}$. am. III 13,27 ; trist. III 13,$14 ; \mathrm{ib} . \mathrm{V} 5,8$. Fast. V 35. ), namentlich zu gottesdienstlichen Zwecken, weshalb es auch Priestertracht ist (Verg. A. X 539. Prop. V (IV) 11, 54 von einer Vestalin; Mopsus bei Val. F1. I 385), und Hor. C. I 35, 21 die Göttin Fides selbst albo velata panno nennt. Bei Schilderung römischer Verhältnisse ist in der Männertracht meist die weiße Toga (toga candida) damit gemeint, vgl. Stat. Silv. V 2, 67; daher es auch die passende Tracht für den Theaterbesuch ist, Mart. XIV 137. I uv. 3, 179; vgl. außerdem noch Ov, am. III 2,41 . Mart. XIV 139. Coripp. Iust.

6) Ieh glaube, daß anf diese Weise das Epitheton albus sich genügend erklären läßt $\mathrm{Lu}$ cas, Quaest. lexilngicae p. 181 meint. Horaz habe das homerisehe Epitheton des Notus cंeysoins (z B. II. XI 306) falsch ïbersetzt: es gehe nicht auf die Farbe, sondern auf die Schnel-

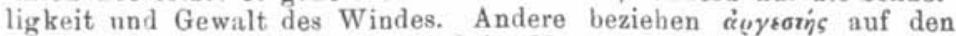
weißen Schaum, den der Südwind im Meere hervorbringt; das Irrige der Ansicht von Lncas beweist aber die Bemerkung des Poseidonius bei

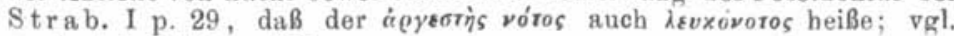
auch ebd. XVII p. 837. 
II 316 und A p. Sid. carm. 23, 324 von Wagenlenkern. Auch die weißen Kopfbinden (vittae) gehören zur festlichen Tracht (Ov ann. III 6, 56; met. II 413; Sil. It. XVI 243), werden aber noch öfter als Abzeichen priesterlicher Würde (Ov. met. II 410 u. XV 676) oder der Seher (Stat. Ach. I 11; Theb. IV 218 u. VI 331) erwähnt. In allen diesen Fällen ist es lediglich die weiße Farbe selbst, auf die es ankommt, ohne daß der Begriff des Glanzes, den weiße Stoffe oft haben, dabei in Betracht käme; und daher kommt denn albus im übrigen als Attribut der Leinwand für gewöhnlich nicht vor, und wenn einmal bei $\mathrm{Ov}$. her. 2, 12 die Segel alba vela heißen, so ist das vereinzelt, da sonst in der dichterischen Sprache auch hierfür candidus stehend ist. - Bei der Bewaffnung wird ein paar mal weiße Farbe des Helmbusches erwähnt, Stat Theb. VI 331. Sil. It. II 399. Bei Vergil A. IX 548 heißt der Schild parma alba; die neueren Erklärer (Servius bietet nichts darüber) fassen das als einen ganz einfachen, nicht mit kunstreichen Arbeiten verzierten Schild, legen aber damit in albus eine Bedeutung, die sich sonst nirgends nachweisen lässt. Das inglorius bei Verg. ist wohl nur durch parma selbst begründet, weil diese der Schild der geringer geachteten Velites ist; albus aber dürfte hier, wie bei Val. Fl. VI 99 , wo albentes parmae genannt sind, sich nur auf die helle Farbe des Leders bezieht, da die parma in der Regel von Leder war. Unverständlich jedoch ist mir, worauf bei Ap. Sid carm. 5, 91: albis os nigrum telis gravidum die Farbenbezeichnung sich bezieht.

Sonst ist albus als Epitheton ornans bei gewerblichen Objekten anderer Art ungemein selten; ich kann nur anführen Pers. 5, 183: fidelia alla, ein weißer Thontopf; Nemes. Cyneg. 153: mulctra, der Melkeimer aus weißem Holz; A uson. XVIII 14, 75, alba pagina, vom P a pier. Ein paarmal kommt der weiße Brettstein vor, P. L. M 15,194 . A. L. 374, 3; bedeutungsvoller ist der weiße Stimmstein, der bekannte freisprechende calculus Minervae, vgl. Ov, met. XV 46. Orest. trag. 944, der sprichwörtlich geworden war, Mart. XI 36, 1 : gemma alba. Sprichwörtlich ist auch alba linea signare, Lucil. frg. 769 (Lachm.), wobei eine weiße Linie deswegen gewählt ist, weil dieselbe auf weißem Papier eben nicht sichtbar ist (also wie wir umgekehrt: „etwas in den Schornstein schreiben“, weil man die schwarze Schrift in dem Ruß der Esse nicht sieht); und ferner Plaut. Pers. I 2, 22: albo rete aliena bona oppugnare, weil ein weißes Netz nicht in die Augen fällt, man also damit jemanden leichter umgarnen kann.

Schließlich haben wir noch die übertragene, auch in Prosa nicht ungewöhnliche Bedeutung von aibus anzuführen, wonach dasselbe für etwas günstiges, glückliches steht, wie umgekehrt ater für unheilvolles, schlechtes; sei es nun, dab diese Be- 
deutung vom glückbringenden weißen Stimmstein herkomme, sei es, was wohl wahrscheinlicher, daß man überhaupt das Helle, Weiße im Gegensatz zum Dunkeln, Schwarzen, als freundlich und segenbringend auffalste. So spricht Hor. Ep. II 2, 189 vom genius albus et ater; Stat. Silv. IV 8, 18 von der alba Atropos. Vgl. ferner Pers. 1, 110: per me sint omnia álba. Mart. X 3, 10: quos rumor alba vehit penna. Sil. It. XV 53: albus dies; dazu auch die schon oben S. 144 angeführten Beispiele.

\section{2) Candidus.}

Es ist schon oben davon die Rede gewesen, welches die eigentliche Bedeutung von candere, candidus ist, und in welcher Weise sich dasselbe von albere, albus, unterscheidet. Bevor wir aber näher darauf eintreten, dies durch Beispiele aus der poetischen Litteratur zu belegen, müssen wir darauf hinweisen, daß die nach verschiedenen Richtungen hin erweiterte Bedeutung dieser Worte von vorn herein den Ausschluß einer ganzen Anzahl von Fällen nothwendig macht. Candere (candor nur äußerst selten) geht bekanntlich aus der Bedeutung von ,weiß glänzen" in die von ,aus Hitze erglühen, glühend heiß sein" über: sei es nun, daß die schon frühzeitig gemachte Beobachtung, daß glühend gemachtes Eisen weiBe Farbe annimmt, zu dieser erweiterten Bedeutung führte, sei es daß überhaupt der lebhafte Glanz, welcher bei glühenden Körpern beobachtet wird, auch ohne Rücksicht auf die weiße Farbe jene Veränderung des Sinnes veranlaßt hat. Da bei derselben aber vielfach der Begriff des Glanzes völlig aufgegeben ist, wie z. B. wenn candere von sommerlicher Hitze, vom Wasser u. a.m. gebraucht wird, so haben wir derartige Fälle hier nicht weiter in Betracht zu ziehen; im einzelnen wird freilich die Unterscheidung oft nicht möglich sein, d. h. wir werden Beispiele anzuführen haben, bei denen eben so gut der Begriff der weißen Farbe oder des strahlenden Glanzes, wie der der Hitze oder Gluth allein angenommen werden kann. Das Adject. candidus hat diesen Wandel der Bedeutung nicht durchgemacht, es wird nie im Sinne von ,glühend heiß" gebraucht; dafür ist es sehr häufig in übertragenem Sinne gebraucht worden, indem der Begriff des Hellen, Heitern auf abstrakte Dinge oder auf menschliche Verhältnisse übertragen wurde und in die Bedeutung einerseits von "glücklich, froh", andererseits von ,klar, offen, wohlgeneigt" u. dgl. überging. Auch diese Fälle haben wir demnach auszuscheiden und ebenso, wo candor im entsprechenden Sinne vorkommt, was gleichfalls sehr gewöhnlich ist, während hier wiederum candere diese übertragenen Bedeutungen nicht erhalten hat.

Was die zum Stamme candere gehörigen, hier in Betracht kommenden Wörter zunächst rein äußerlich, nach der Häufigkeit der Anwendung betrachtet, anlangt, so überwiegt in den von 
uns anzuführenden Fällen, d. h. also denjenigen, bei denen es sich um die Bedeutung von Farbe oder Glanz handelt, weitaus das Adj. candidus. Unter den in runder Zahl 400 Belegstellen, die ich zusammengestellt habe, entfallen etwa 68 Proc. auf candidus, nur 15 Proc. auf das Partic. candens, 10 Proc. auf Umschreibungen mit candor, während andere Formen des Verbums candere nur ganz vereinzelt (etwa 3 Proc.) vorkommen. Von andern Wörtern finden wir candefacere (Pla ut. Most. 259), candescere (in nicht übertragenem Sinne) fünfmal (incandescere einmal, Cat. 64, $13^{7}$ ); candicare, candidare u. a. gar nicht, ausgenommen das Partic. candidatus, Plaut. Rud. 270. Auf gewisse Unterschiede im Gebrauch von candidus und candens werden wir nech hinzuweisen haben.

Candidus bezeichnet im allgemeinen, wie schon gesagt, ein schönes, glänzendes Weiß ${ }^{8}$, und ist als solches eben so dem niger entgegengesetzt, wie albus dem ater; vgl. Lucr. II, 765: curea, quae nigra fuerint paulo ante colore, Marmoreo fieri possint candore repentes. Verg. Eel. 2, 16: quamvis ille niger, quamvis tu ca ndidus esses. Ps. V erg. Dirae 99: candida nigra oculi cernunt. Iuv. 3, 30: qui nigrum in candida vertunt ${ }^{9}$ ). Bisweilen wird es allerdings fast identisch mit albus gebraucht, so z. B. L u c r. II 731: ne forte haec albis ex alba rearis Principiis esse, ante oculos quae candida cernis, wie denn auch der Gebrauch beider Worte in vielen Fällen ganz der gleiche ist; indessen ist es noch eher candens, welches dem albus nahe steht, und daher auch den Gegensatz zu ater bildet, so Lucr. II 771: continuo id fieri candens videatur et album; Ov. met. XI 314: candida de nigris, et de candentibus at ra Qui facere adsuerat. Indessen ist die ursprüngliche $\mathrm{Be}-$ deutung von candere doch wohl nicht die der weißen Farbe, sondern des Leuchtens, resp. des in mehr weißem als rötlichem Lichte Strahlens ${ }^{10}$ ).

Wir beginnen die Aufzählung der Anwendungen von candere, candidus, wiederum mit dem menseh lichen Körper. Wenn wir oben sahen, daB das albere bei demselben eine wirkliche Weiße, d. h. eine kranke, ungesunde Hautfarbe bedeutet, so ist dagegen der candor der $\mathrm{H}$ a ut resp. des $\mathrm{Flei}$ s $\mathrm{ches}$ ein hervorragendes, bei den Dichtern so ungemein häufig angebrachtes Lob von schönen Mädehen, Frauen und J ünglingen,

7) $\mathrm{Ov}$. met. II 728 gehört nicht hierher.

8) Döderlein a. a. O. $194 \mathrm{fg}$.

9) DaB dieser Gegensatz nicht immer festgehalten, sondern bisweilen auch dem albus niger, dem candidus ater entgegengesetzt wird, zeigen u. a die oben S. 144 angefübrten Beispiele.

10) Benfey in Kuhus Zeitschr. VII 59 bringt candere zusammen mit der Wurzel kiand, leuchten; vgl. Curti us a. a. O. S. 522 . Ein Zusammenhang mit canus, canērr, der bisweilen angenommen wird

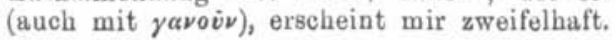


daB mehr als ein Viertel aller in Betracht gezogenen Fälle eben hierauf fallen. Die Candida puella kommt fast bei allen Dichtern vor, vgl. Catull. 13, 4; 35, 8 ; 68,$70 ; 86,1$; Verg. Ecl. 7, 38. Hor. ep. 11, 27 ; Sat. I 2, 123. Ps. Ti b. IV 2, 12. Prop. III 15 (II 22), 8; V (IV) 8, 32; Ov. am. I 7, 7; ib. 7, 40 ; II 7, 5; met. XIII 789. Pers. 3, 110 . Priap. 46, 1. Mart. I 115,$2 ;$ IV 62,2 . Coripp. Iust. II 50. Orest. trag. 57; auch Frauen aus der Heroensage oder Göttinnen werden nicht selten durch dies Beiwort ausgezeichnet, V e r g. Ecl. 2, 46; Aen. V 571; VIII 138; ib 608; Cir. 392; Catal. 11, 27. Prop. II 9, 10; III 21 (II 26) 16; III 26, 5 (IV 28, 51). Ov. am. II 18, 29. P etron. frg. 51, 16. Stat. Silv. IV 8, 29. I uv. 6, 526. Claud. rapt. Pros. I 216; II 18. Dracont. 8,440 . A p. Sid. ep IV 8, 5 v. 12 . P. L. M. 53, 231; und die Dichter lieben es, die strahlende Weiße des schönen Frauenkörpers durch allerlei Vergleich mit andern, durch besondere Weilse sich auszeichnende Dinge, wie Schnee, Lilien u dgl., noch mehr hervorzuheben. Einen Gegensatz zur puella candida bildet sowohl die flava, die Blondine, da diese gewöhnlich lebhaftere Farben hat, wie die fusca, die Brünette, deren Teint gebräunt ist, vgl. Ov. am. II 4, 39: candida me capiet, capiet me flav a puella, Est etiam in $f u s c$ a grata colore venus; ib. III 7, 23: flava Chlide, candida Pitho. Fast. III 493: ut puto praeposita est fuscae mihi candida pelex. Ps. Ov. her. 15, 35: candida si non sum, placuit Cephez̈a Perseo Andromede, patriae fus c a colore suae. In andern Wendungen wird im allgemeinen der candor corporis gepriesen (Plaut. Menaech. 181. Prop. I 2, 19; III 20 (II 25), 41. Claud. epithal. Pall. et Cel. 126. Anth. Lat. 213, 1), die candida membra (Ov. met. II 607. Ps. Ti b. IV 4, 6), die candida forma (Prop. III 27 (II 29), 30; IV 10 (III 11), 16); bisweilen wird auch in malerischer Weise hervorgehoben, daß die wahre Schönheit dieses candor eben darin besteht, daß auch das Blut durch die Haut schimmert, und erst die Verbindung von zartem Weiß und sanfter Röthe den wirklich schönen Teint ergiebt: $\mathrm{Ov}$. am. III 3, 5: candida candorem roseo suffusa rubore; met. X 594: inque puellari corpus candore ruborem traxerat. Dracont. 8, 519: candida sis roseo perfundens membra rubore, (aber in richtiger Mischung, $\mathrm{Cl}$ a ud. nupt. Hon. et Mar. 269: nimio nec sanguine candor abundat); doch kann auch das Blut einer Wunde einen effektvollen Farbengegensatz bewirken, $\mathrm{O}$. met. II 607: candida puniceo perfulit membra cruore, und in Nachahmung Orest. trag. 792: candida puniceo rutilantur membra cruore. - Seltner wird die Schönheit des candor am männlichen Geschlecht gepriesen; am erwachsenen Mann überhaupt nicht, denn für den ziemt sich diese mehr weibische Hautfarbe nicht (vgl. O v. a. a. I 723: candidus in nauta turpis co- 
lor), aber an Knaben und Jünglingen: Verg. Eel. 2, 16. Hor. ep. 3, 9; Ep. II 2, 4 Prop. I 20, 45. Calpurn. ecl. 6, 14. Mart. IV 42, 5. P. L M. 53, 33; auch hier wird daneben das Roth der Gesundheit nicht vergessen: Ps. Tib. III, 4, 21: c andor erat, qualem praefert Latonia Luna, Et color in niveo corpore purpureus. Ov. met. III 423: in niveo mixtum candore ruborem. Dracont. 2, 66: quem $r u b o r$ ut roseus sic c andor lacters ornat; hingegen von blutiger Wunde Sil. I t. IV 204: per candida membra it fumans cruor. - Selbstverständlich ist es vor allem das Gesicht, in welchem sich diese gepriesene Hautfarbe zeigt, daher dieses ganz besonders gern genannt wird, als candida ora (Ov. med. fac. 52 ; met. II 861 . Dracont. 7, 20. A. L. 218,2$)$ oder candor in ore (Ov. a. a. III 227 ; met. IX 787. Prop. IV (III) 24, 8); facies candida (Prop. II 3, 9. Maximian. 4, 7), vultus candidus (Ma rt. VI 39, 12. A. L. 131, 1). Hier vornehmlich sucht die Kokette die von der Natur versagte Farbe durch Schminke zu ersetzen, Ov. a. a. III 199: inducta candorem quaerere creta; und hier ist es auch, wo der Wechsel von Weiß und Roth, nicht bloß von Natur, sondern auch durch vorübergehende Affekte, durch das Erblassen der Furcht oder das Erröthen der Scham hervorgerufen, von besonderem Reize ist, vgl. Stat. Silv. II 1, 41: purpure o suffusus sanguine candor; id. Theb. II 231: candida purpureum fusae super ora ruborem. P. L M. 42, I 35: rubor et candor pingunt tibi vultus. Dracont. 6, 8: candor pallorque ruborque.. qui vernat in ore puellis. Maximian. 1, 89: candida contempsi, nisi quae suffusa rubore Vernarent, propriis ora serena rosis. Orest. trag. 127: permixtus candore rubor pallore fugato $\left.{ }^{11}\right)$. Daß aber das Erblassen der W a ngen, wofür sonst pallere die gewöhnliche Bezeichnung ist, durch candere

11) Ich füge hier noch einige andere Beispiele dieses dichterischen Brauches an, bei denen candere nicht vorkommt, sondern andere Bezeichnungen. Enn. Ann. frg. 355: et simul erubuit ceu lacte et purpura mixta. Ps. Tib. III 4, 30: color in niveo corpore purpurens; ib. 32: inficitur teneras ore rubente genas. $0 \mathrm{v}$. am. [ 8, 35: decet alba quidem pudor ora; ib. III 3, 6: niveo lucet in ore rubor. $\mathrm{P}$ s. 0 v. her. 19 (20), 120: quique subest niven lenis in ore rubor. Se n. Phaedr. 384: ora tingens nitida (Markland: nivea) purpureus rubor. Stat. Theb. I 537: pariter pallorque ruborque Purpureas hausere genas. Ib. XI 336: alternos vultus pallorque ruborque mutat. 1d. Ach. I 161: niveo natat ignis in ore purpureus. Cla ud, epith. Pall. et Cel. 41: niveas infecerat igni Solque pudorque genas. Id. rapt. Pros. 1 271: niveos infecit purpura vultus. D rac o nt. 2, 67: illi purpureo niveo natat ignis in ore ; ib. 8, 499: venit pallente rubore, Nam flammis perfusa genas albentibus ibat: ib. 10,229 : permixto pallore rubens: ib. 13, 9: pallens herba rubet: color est hic semper amantum I $133 \mathrm{M} \mathrm{ax}$ i m i a $\mathrm{n}$. pro niveo rutiloque prius nunc inficit ora Pallor; id. 4, 29: subito inficiens rultum pallorque ruborque. Orest. trag. 524: pallida punicen perfundens ora cruore. Dazu vgl. man das ausfihrliche, an $\mathrm{Hom}$. Il. IV 141 sich anlehnende Gleichniß bei Verg. Aen. XU $66 \mathrm{ff}$. 
wiedergegeben wird, ist selten; s. Senec. Phaedr. 870: ne languido pallore canderent genae. - Weiterhin werden dann bei Mädchen wie bei Jünglingen, wenn auch vornehmlich bei ersteren, gerühmt der glänzend weiße $\mathrm{Hals}$, candida colla (V e r g. Geo. IV 337. Prop. IV 16 (III 17), 29. Ov. a. a. II 457 ; met. IX 388. Claud. carm. $30(48), 11$. Dracont. 2, 85), die Brust (Verg. A. IX 432. Ov. her. 15 (16), 250; ex Pont. II 5, 37. Lucan. X 141), die Schultern (Hor. C. I 2,31 ; ib. 13, 9), die Arme, candida brachia (Prop. III 8 (II 16), 24; III 15 (II 22), 5. Ov. am. III 7, 8. Eleg. in Maec. (P. L. M. 6), 61. Stat. Silv. III 5, 66. Sil. It. III 414) oder candentes lacerti ( $\mathrm{Ti}$ b. I 8, 33), die Hände (Plaut. Pseud. 1262. Stat. Silv. III 4, 59), der Nacken (Hor. C. III 9, 2. Iuv. 10, 345); auch die Weichen, inguina, diese aber nur in Beziehung auf die Skylla, um den Gegensatz zwischen dem zarten Frauenkörper und den häßlichen daran gefügten Hundeleibern recht anschaulich hervortreten zu lassen (Verg. ecl. 6, 75 und Ciris 59: candida succinctam latrantibus inguina monstris. Prop. V (IV) 4, 40: candidaque in saevos inguina versa canes); ferner die Schenkel (Ps. Tib. IV 3, 10. Nemesian. Cyn. 90) und FüBe (Hor. C. IV 1, 27). Zur schärferen Beleuchtung des Weiß dient auch hier bisweilen noch die Hervorhebung bunter, zumal rother Tracht; so Eleg. in Maec. 61: subducere vestem Brachia purpuream candidiora nive. Nemes. Cyneg 90: candida puniceis aptantur crura cothurnis; auch blutiger Wunden, wie $\mathrm{Ps}$. Tib. IV 3, 10: candidaque hamatis crura notare rubis. - Durchmustern wir die zahlreichen von uns angeführten Stellen, so muls eines auffallen: während im allgemeinen, wie wir oben sagten, der Gebrauch von candens gegenüber dem von candidus sich etwa wie 1 : 7 verhält, kommen hier unter 114 Stellen, die sich auf den candor des menschlichen Körpers beziehen, nur zwei Stellen vor, wo anstatt candidus resp. candor das Partic. candens gebraucht ist, nämlich Hor. C. I 2, 31 : candentes humeri, und Tib. I 8, 33: candentes lacerti. Das stimmt zu dem oben von uns Gesagten, daß cundens sich mehr dem albus, dem gewöhnlichen Weiß, nähert, als candidus, wofür wir auch weiterhin noch andere Belege finden werden.

Für die weiBen $\mathrm{H}$ a a re ist candidus beträchtlich seltner als canus, und auch seltner als albus. Wir finden sie sowohl durch candidus bezeichnet (Ps. V e r g. Cir. 121 : candida caesarie tempora. Val. F1. VI 61: candidus crinis. Mart. VII 89,3 candidas. . comas. A u son. XIX 38, 4: caput... candidum. P. L. M. 19, I 46 : candida tempora), als durch candere (P rop. III 10 (II 18), 5: si iam canis aetas mea candeat annis. Ps. Verg. Cir. 320: candentes canos) und candescere (T'i b. I 10, 43: caput candescere canis). Im allgemeinen hat man dabei wohl an silber- 
weißes Haar zu denken; doch ist das nicht in allen Fällen so genau zu nehmen, da wir an verschiedenen Stellen die canities der Haare mit dem candor zusammen verbunden finden. Wenn Ver g. ecl. 1, $27 \mathrm{fg}$. den Tityrus sagen läßt: Libertas; quae sera, tamen respexit inertem, Candidior postquam tondenti barba cadebat, so geht das auf einen ,etwas weißen", also graumelirten Bart eines alternden Mannes ${ }^{12}$ ). - Die $Z$ ähne heißen bei Ca t. 39, 1 candidi dentes, hier mit sehr bestimmter Hindentung, wie der Zusammenhang ergiebt, daß sie glänzend weiß sind, wogegen A. L. 114,8 bei candentes dentes nur an den Gegensatz der weißen Zähne zu den rosea labia gedacht ist. - Daß Gebeine candida genannt werden, ist auch nur vereinzelt, gegenüber der häufigen Anwendung, die wir hierfür bei albus gefunden haben; ich kann nur Ps. Tib. III 2, 10: candidaque ossa super nigra faville teget, und ebd. 17: ossa Incinctae nigra candida veste legent, dafür anführen, wo beide Male der Gegensatz der weißen Knochen gegen die schwarze Asche resp. die schwarzen Trauerkleider den Dichter veranlalst hat, die stärkere Farbenbezeichnung zu wählen.

Sehr häufig ist dagegen, wenn wir nunmehr zur Thierwe l t übergehen, der Gebrauch von candidus für Pferde, zumal (wie bei albus) für Triumphalgespanne; V erg. A III 358: candore nivali; XII 84: qui candore nives anteirent. Ov. met. VIII 373: nive candidioribus equis; ib. XII 77. Sil It. IV 219. Claud. eons. Stilich. II 369; id. VI cons. Honor. 370; ib. 476 u. 507. I 1. Latina 733. Mart. Cape11. II 126. Wie wir gern von schneeweißen Rosen sprechen, so finden wir auch hier mehrfach den Vergleich mit dem Schnee gewählt; ein Unterschied im Gebrauch von candidus und candens liegt jedoch nicht vor, wie ja denn überhaupt der dichterische Sprachgebrauch im allgemeinen der war, daß zwar candidus fast durchweg für schimmerndes Weil und nur ausnahmsweise für Weiß schlechthin oder gar für mattes Wei $\beta$ verwendet wurde, candens dagegen zwar sehr häufig in der letzteren Bedeutung aber nicht minder oft auch ganz mit candidus identisch gebraucht wird. Es folgen die glänzendweißen $\mathrm{Rinder}$, sowohl in Beziehung auf Opfer (Verg. A. IV 61; V 236; IX 628. Ov. met. XII 248 ; trist. IV 2, 5. S e ne c. Agam. 364 ; Oed. 303; Med. 60. Stat. Ach. I 315 ; Theb. VI 865 . In c. Nux 173), als ohne dieselbe (Varr. Sat. Men. p. 146, 4. O v. am. III 5, 10. S t a t. Theb. IX 334. Sil. It. IV 548. Dracont. 8, 418).

12) Servius z. d. St. will allerdings, weil unter der Person des 'Tityrus hier Vergil selbst spreche, candidior nicht auf barba beziehen, da Vergil damals erst 28 Jahre alt gewesen sei (nam XXVIIL annorum barbum quivis potest metere, sed non canam), sondern auf die libertas. Allein die Allegorie geht nicht so weit, daß äberall in der Rolle des Tityrus Vergil zu suchen sei; gerade die cancidion bu'ba entspricht dem Gedanken, daß die libertas sera kam. 
Wenn auch hier der Gebrauch von candidus und candens wechselt, so kann man sich erinnern, daß unter den Rinderheerden Italiens neben blendend weißen auch silbergraue sehr häufig anzutreffen sind. - Nur spärlich sind $\mathrm{S} \mathrm{ch}$ a fe oder Wid de r mit candidus verbunden (L u c r. II 322. Verg. Geo. III 387); ferner haben wir auch hier die a l ba ni s ch e S a u anzuführen, obgleich für diese, der etymologischen Spielerei wegen, albus häufiger ist, vgl. Verg. Aen. VIII 82 . I u v. 12, 72; und wenn I u v. 10,355 von einem candidulus porcus spricht, so liegt darin für den, der nicht an unsere Schweinerace, sondern an die glatten Thierchen im Süden denkt (wie nach einer bekannten Anekdote das Kind ausruft; Ho veduto un piccolo animale nero tutto bianco!), nichts Verwunderliches ${ }^{33}$ ).

Unter den Vögeln steht in erster Linie wiederum der schneeige $\mathrm{S}$ c hw a n, für dessen Gefieder der candor so recht die passende Bezeichnung ist; vgl. Ver g. ecl. 7, 38; Aen. IX 563. Ov. her. 15 (16), 250. Lucil. Aetn. 89. Sil. It. XIII 116. M a rt. I 115, 2. Mart. Capell. IX 918. Wenn bei Germanic. Arat. 465 auch das Sternbild des Schwans candidus cycnus heißt, so kann dabei ebensogut die Farbe des Schwanes selbst, als der Glanz des Gestirnes (vgl, unten) maßgebend gewesen sein. Seltner dagegen ist die Benennung für T a uben (Stat. Theb. XII 20. Dracont. 10, 158) ${ }^{14}$ ); Gänse (Lu cr. IV 681. N eme s. Cyneg. 314. A. L. 406, 2) und Störehe (Verg. Geo. II 320. Ov. met. VI 26) ${ }^{15}$ ); $\mathrm{Cl}$ a ud. in Eutr. I 318 gebraucht candor auch vom sprichwörtlichen wei $B$ en $\mathrm{Rab}$ en, für den albus sicher mehr angebracht ist. Ebenfalls aus später Quelle ist die candens sepia A. L. 295,2 ; es handelt sich dabei um den Gegensatz des Aeußern gegen den schwarzen Saft des Thieres.

In der Pflanzenwelt behaupten ebenfalls die Lili e n den Vorrang, meist candida lilia genannt (V e r g. A. VI 708. Prop. I 20, 38. Ov. met. IV 355 ; ib. V 392. Calpurn. ecl. 3,53 u. 6, 33. Nemes. ecl. 2, 47. A. L. 420, 111. D raco nt. 10,116 ; vgl. M a rt. I 115,3 und die Stellen mit candor, Cl a ud. epith. Pall. et Cer. 126. A. L. 214, 4. Dracont. 6,8$)$, viel seltner candentia (C1 a ud. laus Seren. 90. A. L. 420,38 ; vgl. Nemes. ecl. 4, 22 : nec semper lilia

13) Die weiße Hirschkuh bei Sil. It. XIII 116 ist poetische Erfindung.

14) Wenn bei Stat. Theb. IX 768 die boeotische Stadt Thisbe candida heißt, so ist dabei vielleicht die Taubenzucht, um deren willen der Ort berühmt war (vgl. Ov. met. XI 300. S t a t. Theb. VII 261), die Veranlassung gewesen.

15) Vgl. ferner noch Ps. Verg. Cir. 205 : candida ciris. Wenn A. L. 320,1 vom capo phasianicus es heißt: Candida Phoebeo praefulgunt ora rubore, so scheint hier candidus ausnahmsweise von glänzend rother Farbe gebraucht zu sein.

Philologus. N.F. Bd. II, 1.

Brought to you by | University of Texas at El Paso 
candent. Bei $\mathrm{O}$ v. met. XII 441 lesen die neueren Herausgeber: canentia lilia), was das oben über den Gebrauch von candens $\mathrm{Ge}-$ sagte bestätigt. Neben den Lilien kommen andere Blumen kaum in Frage; die Blüthe des Ligusterstrauch sennen Ov. met. XIII 789. M art. I 115, 3. C la ud. in Eutrop. I 348, sämmtlich für Vergleiche; eine ganze Reihe von Sträuchern finden wir im zehnten Buch des Columella als candida bezeichnet (97 leucoia, 186 lactuca, 254 beta, 396 cucumis, 402 fiscella), den Balsamstrauch bei Stat. Silv. III 2, 141: candida opobalsama; die Weißpa p pel heißt bei Verg. Ecl. 9, 41 candida populus, was neben dem sonst üblichen populus alba eben so gerechtfertigt ist, wie unsere botanische Bezeichnung Silberpappel. - Unter den Früchten sind es die A e pfe l, bei denen einige Male die Weiße gerühmt wird, freilich nur in Verbindung mit der die Reife andeutenden Röthe; so Ov. met. III 483: poma ... candida parte, parte rubent. Ps. Tib. III 4, 34: candida mala rubent. A. L. 408, 10: candida ... sanguine poma rubent.

Im Mineralreich ist zu nennen der Marmor oder sonst weißer Stein (Kalkstein); so Mart. VI 13, 3: candida lygdos, und daher auch candens Paros bei A p. Sid. carm. 22, 140, namentlich aber die daraus gefertigten Gegenstände oder Bauwerke, wie Bildsäulen, A. L. 210,1 ; die weißen Stimmsteine (s. den Abschn. am Ende), A ltäre, Ov. Fast. VI 394. Mart. IX 90,17 ; bei Ov. ex P. III 2, 53 mit malerischem Hinweis auf den Kontrast des darauf vergoBnen rothen Blutes: araque quae fuerat natura candid a saxi, Decolor adfuso tincta cruore $r$ ubet; ferner $\mathrm{H}$ ä $\mathrm{u}$ er und $\mathrm{Ma}$ u ern, vgl. candida tecta, Ov. tr. I 9, 7. Claud. cons. Stilich. II 227 ; area, S t at. Silv. II 2, 89; villa, H o r. ep. 1, 29; moenia, Rutil. Namat. II 63 ; und bei Ov. met. X 595 wieder malerisch: cum super atria velum Candida purpure um simulatas inficit umbras; oder auch $\mathrm{Ort} \mathrm{sehaften,}$ welche auf weißen Kalkfelsen liegen und daher weit ins Land hinein schimmern, wie Anxur, Hor. Sat. 1, 5, 26: saxis late candentibus Anxur, bei M a rt. V 1, 6 direkt candidus Anxur genannt, oder bei Prop. IV 15 (III 16), 3 die candida culmina von Tibur. - Wenn es bei Ps. Ver g. Cir. 102 heißt: candida Thesei Purpureis late ridentia littora conchis, so hat man da wohl nicht, wie oben S. 150 bei albicaus litus an die weiße Meeresbrandung, sondern, worauf der Gegensatz zu den purpurnen Muscheln hindeutet, an weißen U fersand zu denken, wie auch Sil. It. X 205 von candentes arenae spricht; denn es liegt an letzterer Stelle durchaus kein Grund vor, hier candens mit glühend heiß zu übersetzen. - Weiterhin haben wir noch einiges vereinzelte aus diesem Gebiete anzuführen: so das Silber (nur einmal im Vergleich, Mart. I 115, 3; wenn A u s on. Mosell. 231 
den Spiegel candens honor nennt, kann man wohl ïberhaupt an Metallspiegel denken); häufiger das weiß glühende Eisen, L u er. VI 148: candens ferrum, ebenso Ov. Fast. IV 287 ; candens ensis, Verg. A. XII 90 ; chalybs, S il. It. I 171, wobei man allerdings, zumal überall candens steht (das Partic., weil es sich um eine vorübergehende, nicht um eine dauernde Eigenschaft des Eisens handelt), auch bloß die Bedeutung des Glühens oder Erhitztseins annehmen kann. - Endlich kämen auch die beiden weißen Schminken, die Kreide (Ov. a. a. III 199) und das Bleiweiß (M art. IV 25, 2) in Betracht ${ }^{16}$ ).

Unter den $\mathrm{Nat}$ ur produkten nennen wir wiederum zunächst die Wolle („die schimmernde Wolle", bei Schiller), wobei es sich zunächst um das Rohprodukt, nicht um Gewebe handelt (Catu 11.64, 318. Calpurn. ecl. 5, 71, wo fusca lana den Gegensatz bildet; Sen. lud. Claud. 4 v. 5. Stat. Silv. I 4, 123); ferner die Mileh (Luer. I 258. Varr. Sat. Menipp. p. 102,1 ; ib. 145, 4. Ov. her. 15 (16), 249 ; ex Pont. II 5, 37), in der Regel zu Vergleichen benutzt, wobei wohl auch hervorgehoben wird, daß es sich um frischgemolkene Milch handelt, die ja in der That weißer ist, als abgestandene (O v. am. III 5, 13: candidior, quod adhuc spumis stridentibus albet, Et modo siccatam, lacte, relinquit ovem); auch Käse (O v. Fast. IV 371) und das Weiße des Eies (Mart. XIII 40, 1. Seren. S a m m. 764; 1043; 1047). Wenn Ov. met. VIII, 677 die Wabe candidus favus nennt, so ist dabei wohl nicht das weißliche Wachs gemeint, sondern der darin enthaltende, weiß schimmernde Honig selbst, der bei Ov. Fast. III 762 candida mella heißt ( $\mathrm{O}$ v. ebd. I 186 liest Peter mit einigen Hss.: et data sub niveo candida mella cado, dagegen Merkel mit anderen condita. Vielleicht ist hier auch anst. sub cado, was mir wenig passend erscheinen will, sub favo zu lesen). Da es in der That Honig giebt, weleher weißliche Farbe hat, so kann man auch hier neben der Bedeutung des Schimmerns noch die der weißen Farbe beibehalten; sonst ist allerdings flavus das gewöhnlichere Attribut für den goldgelben Honig. - Das W e i B brot heiBt in Prosa häufig panis candidus; in der Poesie habe ich nur das späte candens quadra gefunden, A. L. 291, 6 . Für den weißen Wein wird candidus nicht gebraucht, doch hat Tib. I 5, 24 cándida musta, was auf Most von weißem Weine geht, vgl. Plin. XXIII 29: musta differentias habent naturalis has, quod sunt candida aut nigra aut inter utrumque. - Sehr bezeichnend ist candidus für das Elfen bein oder für daraus gefertigte Gegenstände (Ca t u 11. 64, 45. V e r g. A. VI 895. Ovv. ex P. III 3,98$)$; und da, wo das Material nicht angegeben ist

16) Welchen Edelstein Prisc. carm. 2, 855 mit den Worten : gemmaque, quae radius emittit candida solis, meint, weiß ich nicht zu sagen; vielleicht den Diamant, event. den Opal. 
und der Gegenstand nur candidus genannt wird, wie Bettf ü B e (C a t. 61, 111), die G riffe (cornua) einer Bücherrolle (Ov. tr. I, 1, 8), ein Plectrum (M a rt. XIV 167, wo allerdings manche Handschr. garrula lesen), hat man sicherlich an Elfenbein zu denken. - Endlich sind noch die Perlen hier anzuführen (Sil. It. XII 66. Cla u d. VI cons. Honor. 528).

Vom W a s ser wird albus, wie wir gesehen haben, nur gebraucht, wenn dasselbe im Zustande des S chaumes oder durch $\mathrm{Seh}$ wefelbestandtheile weißlich ist. Beide Fälle kommen auch hier in Betracht; für Schaum der Wellen, wenn auch nicht candidus, so doch candere (Lucr. II 767 : vertitur in canos candenti marmore fluctus) und incandescere (C a t. 64, 13 : spumis incanduit unda); und der schwefelhaltige Nar heißt bei M a rt. VII 93, 1 candidus amnis. Indessen wird candidus doch noch in weiterem Sinne gebraucht, als albus, indem es von ganz klarem, farblosem und durehsichtigem Wasser gesagt wird. Diese Bedeutung hat es offenbar bei Mart. VI 42, 19: quae tam candida, tam serena lucet, Ut nullas ibi suspiceris undas Et credas vacuam nitere lygdon. Bei V a l. F 1. IV 97: (Sol) traxit diem candentibus undis, kann man freilich eher daran denken, daß der Augenblick gemeint ist, wo die Sonne zuerst voll das Meer bescheint, da hierfür der Ausdruck candere auch sonst gebräuchlich ist; vgl. die Beispiele unten und $\mathrm{Enn}$. trag. frg. 332 (Vahl.): lumine . . terra et cava caerula candent.

Ungemein hänfig ist candidus als Attribut für Schnee und $\mathrm{E}$ is. In den meisten Fällen handelt es sich da wieder um Vergleiche, indem Kleider, Pferde, Frauenkörper u. dgl. als weißer denn Schnee gepriesen werden; so C at. 80, 2. Verg. A. XII 84. Eleg. in Ma e e n. 62. Ov. am. III 5, 11; ib. 7,8 ; her. 15 (16), 249 ; met. VIII, 373 ; ex Pont. II 5, 38. Sil. It. XIII 116. Mart. I 115,3 ; IV 42,5 ; VII 33,2 ; XII 82, 7. Auson. IV 5, 6. Cla ud. epith. Pall. et Cel. 126. Coripp. Iust. I 328 ; doch kommt in einzelnen Fällen

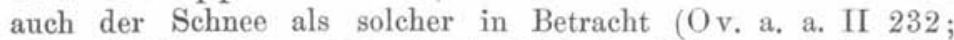
trist. III 10,10 u. 22. A. L. 107, 1. Cla ud. bell. Poll. 345 ) oder die damit bedeckten Berge und Länder (H o r. C. I 9,1 ; III 25, 10. Senec. Here. Oet. 1052. German. Arat. 584. S t a t. Theb. IV 290). Ausdrücklich wird bisweilen hervorgehoben, daß der Dichter ganz frisch gefallenen Schnee meint, der sich seine jungfräuliche Weiße noch bewahrt hat, $\mathrm{O}$ v. am. III 5, 11: candidior nivibus, tunc cum cecidere recentes; ex P. II 5, 38: non calcata candidiore nive. Wenn hierbei unter den 26 Fällen, die wir aufgezählt, sich nnr dreimal candens findet (S t a t. Theb. IV 290. A. L. 107, 1 und Cl a u d. bell. Poll. 345), sonst immer candidus resp. candor, so entspricht das zwar ungefähr dem Verhältniß von $1: 7$, das wir oben im allgemeinen für candidus und candens constatirt haben, verdient aber 
immerhin, da es sich um einen so ausgesprochen weißen und schimmernden Gegenstand, wie Schnee, handelt, Beachtung. Für den $\mathrm{Re}$ if (sonst meist cana pruina) findet sich candere nur in späten Stellen (Avian. fab. 34, 7. Claud. VI cons. Hon. 476 ; epist. 2, 15 . M a r t. Cap. II 116).

Sodann ist ebenfalls sehr oft angewandt, und zwar schon in unsern frühesten Quellen, candere und candidus für die Sonne und deren Glanz. Es ist bald der Himmelskörper selbst oder das von ihm ausgehende Licht, welches so bezeichnet wird (Enn. trag. frg. 318 Vahl.: candentem in coelo facem; ib. 367: hoc lumen candidum; ib. 402: hoc sublimen candens; Ann. frg. 93 : candida lux. Naev. frg. 51 Ribb.: solis candor. Lucr. V 282 ; ib. 1195. Ps. Ver g. Culex 43. Ov. met. VI 49. Ps. Tib. IV 1, 65. A. L. 139, 3), bald der personificirte Sonnengott, Sol oder Phoebus selbst oder sein Wagen (Enn. Ann. frg. 548: radiis rota candida. A t ti us frg. 518 Ribb.: Sol . . candido curru. Incert. trag. 183 Ribb.: qui per caelum candidus equites. Verg. A. VIII 728: candentis Phoebi. Ov. met. XV 30. Val. Fl. III 559. A. L. 139, 43. Coripp. Ioh. II 158 u. III 26), bald die vom Sonnenlicht beleuchteten Gegenstände (Pacuv. frg. 88 Ribb. : terra . . solis exortu capessit candorem; Enn. trag. frg. 332 , s, oben S. 163). Wie wir diesen candor solis zu verstehen haben, das zeigt am deutlichsten Ov. met. XV 193: mane rubet, terraque rubet cum conditur ima : candidus in summo est. Es ist also nicht der rothe Schein der auf-oder untergehenden Sonne, sondern der blendende, eigentlich farblose Schimmer des Tagesgestirns, welcher durch candor gekennzeichnet wird. Ebenso bekommt das Licht ïberhaupt oder helle, klare Luft diese Bezeichnung (P laut. Amphitr. 547. Lucr. IV $338 ; \mathrm{V}$ 776. Stat. Silv. III 1, 71. Rut. Nam. I 197) und schöne, sonnenhelle $\mathrm{T}$ a ge $(\mathrm{Ov}$. her. 15 (16), 318; trist. II 142; Fast. I 637; V 548. Petron. frg. 41, 2), diese dann auch im übertragenen Sinne von glücklichen, ungetrübten Tagen (candidi soles, Catull. 8, 3). Wenn bei Hor. C. III 7, 1 auch der Favonius candidus genannt wird, so ist (wie oben beim Iapyx und Notus, s. S. 152 fg.) die aufheiternde Wirkung des Windes dabei der Grundgedanke. - Aber anch die andern Himmelskörper, die nicht den überwältigenden Glanz der Sonne, sondern milderen Schimmer haben, werden candida genannt; so der Mond (Verg. Aen. VII 8; Ciris 37. Ov. met. IV 332. Petron. sat. 89 v. 54) und sehr häufig die Sterne (Plaut. Rud. 3. Lucr. V 1208. Cic. Arat. $174 ; 248 ; 249 ; 410$. Cic. ap. Prisc. II p. 105, 9. H or. C. III 15, 6. Verg. Geo. I 217. Manil. Astr. I 322 ; ib. 703; 711; 715; 756; $802 ;$ V 217. German. Arat. $41 ; 203 ; 233 ; 480$. S enec. Phaedr. 340. Val. Fl. VII 22 ; von der Milehstraße O v. met. I 169). Der Begriff des Weißschimmerns bleibt auch hierbei sicherlich be- 
stehen; wir, die wir kein dem candor entsprechendes Wort haben, sprechen daher gern vom silbernen Mond und Sternen und geben damit denselben Eindruck wieder, den der Römer durch candidus bezeichnen will. - Hingegen erscheint candidus für den mehr röthlichen Schimmer einer Flamme sehr wenig passend. Wenn bei Enn. Ann. frg. 157 es heißt: prodinunt famuli : tum candida lumina lucent, so kann man allerdings wohl nur an Fackeln denken; es heißt also da ,schimmernd" schlechtweg, eine Bedeutung, die für candere gerade in der ältern Poesie gewöhnlich gewesen zu sein scheint, wenigstens darnach zu urtheilen, daß wir es bei Ennius nur in diesem Sinne, und nicht weniger als siebenmal, dazu in den Fragmenten anderer älterer Tragiker viermal, ebenfalls im Sinne des Schimmerns, finden: eine Thatsache, die man wohl darauf hindeuten darf, daß diese, wie wir oben angenommen haben ursprüngliche Bedeutung des Wortes gegenüber der abgeleiteten Bedeutung "weiß sein" in der ältern Poesie noch ïberwog. Wenn es aber bei Val. Fl. VIII 247 heißt: sed neque se pingues tum candida flamma per auras Explicuit nec tura videt concordia Mopsus, vom Vermählungsopfer des Jason und der Medea, so möchte ich hier, mit Rücksicht auf den Zusammenhang, cundidus nicht als Bezeichnung des farbigen Glanzes fassen, sondern eher im Sinne von "rein" oder "ungetrübt", wie man ja auch von einer vox candida spricht, Plin. XXVIII 58, oder von omina candida, glückverheißenden, Prop. V (IV) 1, 67 u. dgl. Die Flamme kann sich in der dicken Luft (pingues aurae) nicht hell und klar entwickeln, und dies giebt ein ungünstiges Vorzeichen für die Ehe ab.

Unter den gewerblichen Produkten ist es selbstverständlich abermals die $\mathrm{Tracht}$, und zwar wesentlich die festliche und die priesterliche weiße Kleidung, auf welche die Mehrzahl der Belegstellen entfällt; vgl. candida vestis, toga, relamina u. ä., Plaut. Casin. IV 1,9. Titin. frg 167 Ribb. Cat. 64, 308. Ps. Verg. Cul. 130. Ov. her. 4, 71; 10, 41. Val. F1. III 432. Stat. Silv. II 7, 10; Theb. VII 654 . Dracont. 8,617 . Coripp. Ioh. I 260; Iust. II 117; auch eandida (Neutr. plur.) bedeutet weiße Gewänder, M a r t. II 46, 5 ; VIII 28, 16. Wie albus, so wird auch candidus oft im Sinne von weißgekleidet (das prosaische candidatus nur bei Plaut. Rud. 270) gebraucht, Mart. IV 2, 4. Coripp. Iust. II 101; candida Roma, Mart. VIII 65, 6; exercitus, $\mathrm{Cl}$ aud. nupt. Hon. et Mar. 295; curia, id. in Eutr. I 308 ; namentlich candida turba, Tib. II 1, 16. Ov. Fast. II 654; ib. IV 906. Coripp. Iust. III 161. - Auch weiße B in de n, infulae (Lucan. II 355; V 144) und Decken (Hor. S. II 6, 103) gehören hierher. Im allgemeinen hat man bei der Tracht $u$. dgl. sowohl an wollene, wie an linnene Stoffe zu denken, obgleich, wo es sich um römische Tracht oder um Binden handelt, durchschnittlich Wolle 
als Stoff anzunehmen ist; aber auch der ,s chneeige Lein", für den albus, wie wir erwähnten, eine ungewöhnliche Bezeichnung ist, wird gern in seiner schimmernden Weiße durch candere bezeichnet, sei es nun, daß es sich um leinene $\mathrm{T}$ ü ch er oder Kleider (Mart. XII 82, 7. Stat. Silv. I 6, 31. Val. F1. VI 225 ; vgl. Grat. Cyneg. 44), um Zelte (Ov. met. VIII 43. Val. F1. II 447. Cor. Ioh. II 273) oder um Segel handelt (Ca i. 64, 235. Prop. I 17, 26 Ov. a. a. II 6; Fast. V 162. Lucil. Aetn. 585. Val. Fl. I 381. Orest, trag. 43 Für Schuhwerk, das ja nur selten aus weißem Leder gefertigt wurde, kommt candidus nur einmal vor, Mart. VII 33, 2.

Von sonstigen Dingen haben wir endlich noch anzuführen K rysta 11 oder Glas, welches um des Gegensatzes zu dem darin enthalteneu rothen Weine willen candidus heißt bei M a r t. VIII 77, 5: candida nigrescant vetulo crystalla Falerno, und, wie bei albus, den weißen Spielstein (A. L. 372, 2) und Stimmstein (Varr. Sat. Men. p. 165, 9. Pers. 2, 2. Mart. XH 34,7 ); hier kommt denn der glückverheißende Sinn der weißen Farbe hinzu, sodaB halb in eigentlicher Bedeutung, halb in übertragenem Sinne Ov. met. XV 47 von einer candida sententia sprechen kann und $\mathrm{Catu} 11.68,148$ von einem lapis candidior.

Zürich.

(F. f.)

H. Blümner.

\section{Ad Orionem Thebanum.}

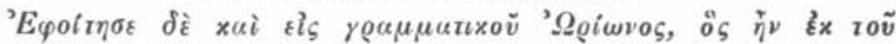

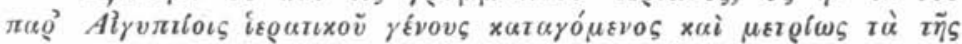

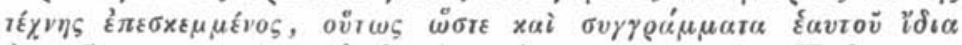

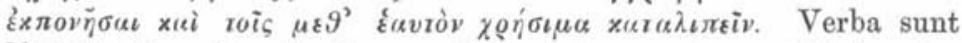
Marini (de vita Procli, cap. 8) aperte mendosa. Istud enim

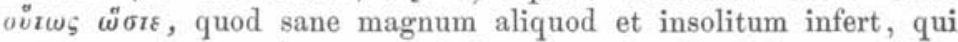
potest stare antecedente vocabulo $\mu$ r Mützellius (de emend. Theog. Hesiod. p. 321**) verba inde ab ô

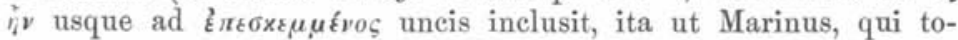
tus versatur in Procli sui laudibus, putide sane et simul brevitate usus ab ipso alienissima dicat: Audivit Proclus Orionem tam studiose, ut vel ipse proprios commentarios scriberet utilesque eos posteris relinqueret. Iure hoc repudiavit Ritschelius (op. I 590), ipse tamen, quod miror, ab offensione nimis tutus. Nam scri-

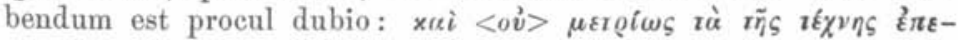

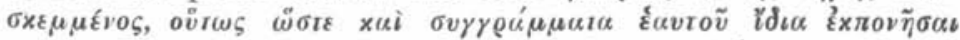

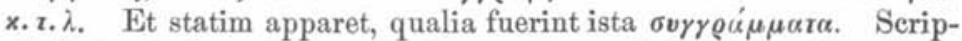
sit Orio nimirum libros, quales novimus Galeni $\pi \varepsilon \varrho i ~ \tau \tilde{\eta} \varsigma ~ \tau \alpha ́ \xi \varepsilon \omega \varsigma$

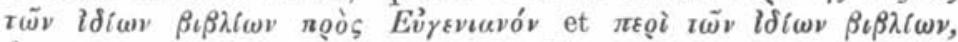
de quibus audi docte disputantem Ilbergium in mus. Rhen. vol.

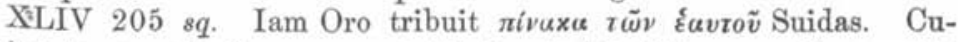
ius tamen schedas cum manifestis turbis laborare satis constet, nullus ego dubito, quin opus illud sit Oro demendum, attribuendum Orioni. Quod potest fieri sine ullo quaestionis Ritschelianae damno.

Lipsiae. Brought to you by | University of Texas at EPPaSmmizch. 